\title{
Mediastinal staging in lung cancer: a rational approach
}

\author{
L. Ceron, L. Michieletto, A. Zamperlin
}

ABSTRACT: Mediastinal staging in lung cancer: a rational approach. L. Ceron, L. Michieletto, A. Zamperlin.

Mediastinal staging is generally performed by the use of imaging techniques such as CT (Computed Tomography) and PET (Positron Emission Tomography), mini-invasive techniques, as TBNA (Transbronchial Needle Aspiration), EBUS-TBNA (Ultrasound-Guided Transbronchial Needle Aspiration), EUS-FNA (Endoscopic Ultra Sound Fine-Needle Aspiration), and/or surgical techniques as mediastinoscopy, thoracoscopy, mediastinothomy.
Each of these techniques provides different sensitivity, specificity and predictive value: all these characteristics need to be well considered and adequately used to achieve the best possible outcome, best exploitation of available resources and least discomfort for the patient. Particularly, indicators which may suggest the need for further examination of mediastinum, following a negative CT and PET, will be discussed in this review; need for surgical confirmation after negative TBNA will be considered, also. Monaldi Arch Chest Dis 2009; 71: 4, 170-175.

Keywords: Transbronchial Needle Aspiration, Esophageal Needle Aspiration, PET-CT, Bayes' theorem.

Pneumology Unit, Thoracic Department, Mestre-Venice, Italy.

Correspondence: Dr. Loris Ceron, Pulmonology Unit, Ospedale dell'Angelo, Via Paccagnella 11, 30174 Mestre-Venice, Italy; e-mail:loris.ceron@ulss12.ve.it

\section{Introduction}

Nowadays, several techniques are available and may be adopted to approach mediastinal staging. Apart from the surgical approach (e.g.: mediastinoscopy, mediastinothomy, thoracoscopy), which is not analysed in this paper, the current available techniques include: contrast-enhanced CT, PET, TBNA, EBUS-TBNA, and EUS-FNA. Some of these, i.e. PET and ultrasound-guided needle aspiration, provide very sensitive and specific results. TBNA alone, on the other hand, although highly specific, has not proved to be sufficiently sensitive and may provide false negative results.

However, in clinical practice and in each particular case, the predictive value of a test should always be considered as the prominent issue by the practitioner, compared to the test's sensitivity and specificity. In other words, probability that a positive/negative result of TBNA or PET will actually coincide with the presence/absence of lymph node metastasis is the real focus (from the clinical point of view) when approaching mediastinal staging. It is well known how two equally sensitive and specific examinations may have different predictive value (positive - PPV and negative - NPV), depending on the expected prevalence of the phenomenon being examined (pre-test probability; in our case: probability of mediastinal lymph node metastasis).

Every pulmonologist's purpose is to rule out the presence of mediastinal metastasis by appro- priate use of available diagnostic investigations. However, no diagnostic path can eradicate the probability of neoplastic findings at surgical exploration; therefore, the real goal is to reduce the risk to the minimum.

With this in mind, the following paragraphs will highlight the best utilisation of the above mentioned techniques. Some relevant features of each of them will be illustrated below; the aim of this analysis is to suggest a reasoned model, based on evidence, for $\mathrm{N}$ factor mediastinal staging in non small cell lung cancer (NSCLC).

\section{Available techniques}

\section{Computed Tomography (CT)}

Besides standard chest $\mathrm{x}$-ray, CT is presently considered the fundamental preliminary examination when evaluating lung cancer. Literature reports rather good specificity of CT (about 80\%) and moderate sensitivity (not above 60\%) [1]. From a clinical point of view, this may infer that one enlarged mediastinal lymph node (short axis $\geq 1 \mathrm{~cm}$ ) in a patient affected by lung cancer may result negative for metastasis in 4 cases out of 10 , whereas identification of metastasis may be achieved in up to $20 \%$ of normal size lymph nodes ( hort axis $<1 \mathrm{~cm}$ ). We may conclude that although a significant difference in average diameter statistically exists amongst healthy and metastatic lymph nodes, secondary neoplastic localisation 
may not be diagnosed based uniquely on this factor [2]. Accordingly, recent ACCP guidelines on mediastinal staging report that $\mathrm{CT}$ may be considered sufficient only in case of massive mediastinal invasion; in any other case, further diagnostic techniques should be implemented [3].

\section{Positrons Emission Tomography (PET)}

PET is probably the most revolutionary diagnostic technique of the last 20 years, when used to investigate NSCLC [4-6]. Similar to other revolutionary instruments now available in clinical practice, PET has encountered fervent supporters and committed opponents. Literature reports excellent sensitivity (between $75 \%$ and $91 \%$ ) and specificity (between $78 \%$ and $93 \%$ ) of this technique when applied to the investigation of mediastinum; generally, results have proven less specific in cases of enlarged lymph nodes and, conversely, less sensitive in cases of normally sized lymph nodes [7].

Although we may consider that the amount of reported false negatives is as low as $5-8 \%$, a recent meta-analysis has shown that this should be trusted when there is a presence of enlarged lymph nodes, whereas, in case of normally-sized lymph nodes, false negatives may reach $25 \%$ (1 out of 4) [7].

As a matter of fact, the above mentioned data may be of no real worth unless translated into effective predictive values. We have mentioned before that prevalence, or "pre-test probability" of a particular event (such as presence of N2 metastatic lymph nodes) should be considered a key factor that affects the predictive value of the examination, which in turn is the only reference parameter whenever assessing an instrumental result.

That said, we may now consider the case of a small peripheral cancer, less than $1 \mathrm{~cm}$ in size: it is accepted that it may cause mediastinal metastasis in about $2 \%$ of cases [8]. Should PET show positive results in mediastinum, the estimated probability of the positive result proving true is 1 out of 10 (since PPV 7-13\%, depending on diameter of lymph nodes). On the contrary, should PET show negative results, the absence of metastasis may be accepted as virtually true (since VPN 99\%).

We shall now analyse a totally different case: a malignant adenocarcinoma clinically staged N1. In such cases, probability of mediastinal metastasis may reach $60 \%$ [9]; PET positivity of mediastinal lymph nodes may exclude doubt about presence of metastasis (since, PPV 87\%), so bioptic confirmation might be considered unnecessary; on the other hand though, negative result to PET could prove wrong 3 times out of 10 (since NPV 71\%), and therefore requires confirmation $[10,11]$. A similar conclusion has been accepted by the previously cited ACCP 2007 guidelines, according to which, in clinical stage I NSCLC positivity to PET of mediastinum requires bioptical confirmation, since PPV of PET (and therefore presence-ratio of me- diastinal metastasis), is unacceptably low in this case. On the contrary, negativity to PET paves way to use of surgery with no need for further examinations $[3,12]$.

\section{Transbronchial Needle Aspiration (TBNA)}

The use of TBNA has been steadily increasing in recent years, so this technique is now well known to pulmonologists and practiced by several endoscopists. Most qualified contributions present in literature indicate values of about $76 \%$ sensitivity and $98 \%$ specificity [13]. As with every other needle-aspiration technique, a positive result may be well considered to be completely trustworthy when staging a tumour; neo-adjuvant chemo therapy generally being the first therapy adopted to tackle a metastatic mediastinal lymph node.

Given the unsatisfactory sensitivity of TBNA, some difficulties may raise when assessing a negative result (that means prevalence of lymphocytes and macrophages on the microscope slide, while an inadequate sample provides no evidence/indicators of a pierced lymph node or neoplasm).

ACCP guidelines suggest that a negative result after needle aspiration must lead to surgical sampling [3]. Nevertheless, with reference to TBNA, we should try to consider the predictive criteria as we did with PET. Going back to the previous illustrative case of stage II ADK (where, as we mentioned, the probability of mediastinal metastasis reaches $60 \%$ ), a positive result to TBNA of a mediastinal lymph node may indicate, with actual no doubt, a secondary localisation (PPV 94\%). On the other hand, a negative result will very likely be a false negative (NPV 54\%, 1 out of 2); intuitively, further decisions should not be based on this negative datum.

We should however picture the situation of a small peripheral tumour less than $1 \mathrm{~cm}$ in size. Notwithstanding the presence of enlarged lymph nodes, the probability of mediastinal metastasis does not exceed 3-4\%. Negative result of TBNA would practically guarantee the absence of metastasis (NPV 99\%), and represent a reliable reference in the staging of mediastinum and in the resulting decisional process. In other words, a negative result after needle aspiration, similarly to PET or to all other techniques, may not be completely interpreted on its own but should be considered within the case history and referred to the predictive value of that specific test in each specific situation.

Accuracy of TBNA may improve when supported by rapid examination of samples (ROSE) [14]. ROSE may be carried out by the same operator performing TBNA; he/she may, with little training, learn to identify an "adequate and diagnostic sample" from a "non adequate" and/or an "adequate - non diagnostic" sample (normal lymph node). In our experience, the implementation of ROSE resulted in $14 \%$ comprehensive reliability improvement of TBNA (personal data - not published). 
Transbronchial or transoesophageal ultrasoundguided needle aspiration( EBUS, EUS)

TBNA yield may improve when supported by other complementary techniques such as endobronchial ultrasound. Published data on this issue concurs that TBNA sensitivity may reach $80-85 \%$ when supported by ultrasound with the use of radial probe and even $>90 \%$ when "convex" real time-probe is used (which allows direct observation of needle piercing of the lymph node) [15-19]. In clinical practice however, worthwhile application of ultrasound appears limited to lymph nodes of 1 and 2 ATS stations (in such cases endoscopists have no definite safe landmarks for the puncture), or to small lymph nodes (5 mm or less) and whenever rapid on site examination is not performed $[20,21]$.

Similar to TBNA, ultrasound guide provides high sensitivity and specificity to trans oesophageal needle aspiration as well (EUS-FNA), although values of sensitivity decrease sensibly when lymph nodes are not visible in the CT images $[22,23]$. Combining both techniques (endobronchial and endoesophageal ultrasound, the so called "medical mediastinoscopy") allows further gain in diagnostic accuracy $[24,25]$.

\section{The healthy mediastinum}

It is well known how the presence of pathological lymph nodes in the hilar site (N1), with no visible anomalies of the mediastinum (stage cII), means high risk of secondary N2 localisation. In a recent study, carried out on 1782 patients which were diagnosed clinical stage II with CT only, the presence of mediastinal metastasis was found in $30 \%(28 \% \mathrm{~N} 2,2 \% \mathrm{~N} 3)$ of patients who underwent surgery [26]. Data published by Cerfolio and colleagues substantially confirm such conclusions in patients who had been staged N1 with CT and PET and who where eventually diagnosed with presence of mediastinal metastasis after a subsequent mediastinoscopy (17\% of cases) or EUS-FNA (23\% of cases) [27]. In both situations, ADK histology generally indicated a higher risk of mediastinal localisations.

Should a patient be staged NO with CT and PET, surgery is usually elected immediately. Systematic use of mediastinoscopy and EUS-FNA on patients staged N0 has shown that presence of mediastinal metastasis generally occurs in 2.7-3\% of cases, therefore inferring that in such situation systematic use of these techniques is not recommended [27, 28]. However, should we make a selection amongst patients staged N0 and perform mediastinoscopy on those affected with right upper lobe tumours, unexpected N2s raise to $10 \%$. Similarly, Cerfolio has found that N2s rise to $15 \%$ if EUSFNA is practiced only on patients affected with right lower lobe tumours. This outcome was partially related to some "risk factors", like the presence of an unfavourable G3 grading, T SUV $>10$ and $\mathrm{T}$ large size [27]. Mediastinal spreading is more likely as well in peripheral ADK involving the pleura or when CEA is elevated [29, 30].

Cerfolio's results may also be explained by considering that mediastinal metastasis follow preferential diffusion paths depending on T's position within lobes; tumours affecting superior lobes have a particular tendency to spread throughout paratracheal and subcarinal lymph nodes, whereas those affecting lower lobes tend to spread throughout the subcarinal site, the para oesophageal sites and the pulmonary ligament (posterior-mediastinum) [31, 32]. We may therefore reasonably apply TBNA and mediastinoscopy in case of tumours affecting upper lobes, these techniques allowing exploration of middle and upper mediastinum; in the case of a tumour of the lower lobes, EUS-FNA may result more effectively, allowing exploration of posterior mediastinum.

\section{The statistical support}

At this point, a diagnostic protocol to approach the mediastinum may be suggested, based on the remarks above; the protocol should develop the best possible exploitation of each specific examination and take advantage of their optimal matching by resorting to statistical analysis. A mathematical model which enables us to predict the probability of nodal metastasis after a certain number of diagnostic procedures has been performed is Bayes' theorem. It moves from pre-test probability of disease and evaluates every test result in terms of likelihood ratio (LR), in order to calculate the post-test probability of disease (table 1); so it allows to obtain a quantitative, and therefore objective, measure of the value added by a diagnostic test (such as a laboratory finding or a radiological test) in the estimation of the probability that some event occurs. This probability is calculated by multiplying Odds by every LR test. Bayes' theorem is already used in the diagnostic approach to the solitary pulmonary nodule, but it fits perfectly in the evaluation of the N2 involvement in lung cancer as well. Indeed it allows the estimation of post-test probability of mediastinal metastasis after the diagnostic path is concluded, giving us a simple way to evaluate when a patient, on the basis of the final probability, could undergo surgery or, conversely, further investigations are required.

Table 2 provides a simplified algorithm focused on a reasoned study of mediastinum with the use of probability calculation; "pre-test" probabilities of metastasis and predictive values of each examination (in case of positive or negative result) are taken into account for each indicated choice. Surgery is arbitrarily considered a possible choice whenever probability of mediastinal metastasis falls below $10 \%$.

Noteworthy, on path $a$ (pre-test probability $<20 \%$ ) a negative PET indicates that there should be surgery directly without mediastinoscopy, and PET itself could be unnecessary in the event of CT negative mediastinum (lymph node size $<1 \mathrm{~cm}$ ). 
Table 1. - Definition of Odds, Likelihood Ratio and Bayes' Theorem

\section{Bayes' theorem (probability theory)}

Mathematical theorem which allows us to obtain conclusions regarding the probability that an event will take place, starting with "pre-test" probability (before any test is used), to formulate a "post-test" probability.

\section{Post-test probability $=\frac{{ }^{*} \text { Odds } x^{*} \text { LR1 } \times \text { LR2 } \times \text { LR3 ... }}{1+(\text { Odds } \times \text { LR1 } \times \text { LR2 } \times \text { LR3...) }} \times 100$}

\section{*Odds}

Ratio between occurrence and non-occurrence rate of some event; that is: ratio between probability of an event and probability of the complementary event.

$$
\text { Odds }=\frac{P}{(1-P)}
$$

\section{*LR (Likelihood ratio)}

Accuracy index of a test, which combines test results among ill and healthy subjects. Likelihood ratio of a positive result: ratio between positive result percentage among affected subjects and positive result percentage among non affected (healthy) subjects. Likelihood ratio of a negative result: ratio between negative result percentage among affected patients and negative result percentage among non affected patients.

Positive or negative likelihood ratio comes from sensitivity and specificity values according with the following formulae:

$$
\begin{aligned}
& L R+=\text { sensitivity/(1-specificity }) \\
& L R-=(1-\text { sensitivity }) / \text { specificity }
\end{aligned}
$$

On path $b$ (pre-test probability $30-40 \%$ ), negative FNA allows surgery when either CT or PET is negative, while FNA could be avoided when both CT and PET are negative; conversely, a negative PET requires FNA if mediastinal nodes are enlarged. On path $c$ (clinical N1 involvement without evidence of mediastinal lymph node enlargement), PET negative mediastinum is not enough to exclude mediastinal metastasis, so direct surgery without mediastinoscopy is a reasonable choice only when both PET and FNA are negative.

In this model therefore, unlike suggested in the ACCP 2007 guidelines, a negative FNA does not require surgical confirmation in any case, but only when probability of mediastinal metastasis is still too high (i.e. $>10 \%$ ) after FNA is performed (posttest probability); a negative PET conversely, although highly predictive of healthy mediastinum, can require in some instances a cytological/histological confirmation.

\section{Conclusions}

Mediastinal staging is a crucial moment to consider the best therapeutic options for a patient; image methods (CT, PET ) provide a fundamental advantage when balanced with their predictive values. Diagnosed stage N0 after CT and PET authorises surgery, except in cases of unfavourable grading, large or central tumours or a very high SUV of the primary tumour. A stage $\mathrm{N} 1$ after CT and PET suggests instead to resort to mini-invasive and/or surgical biopsy techniques, given the major possibility of metastasis in mediastinal lymph node even in case of negative CT and PET. Mini-invasive biopsy methods (TBNA) are precious and conclusive when they give a positive result, while a negative one is not considered final since micro-metastasis remains a risk and plagued lymph nodes may be situated next to unaffected ones. A negative needle aspiration generally compels to adopt surgical confirmation (mediastinoscopy, thoracoscopy), nevertheless diagnostic surgery may be avoided in case of low "post test" probability of neoplastic localisation.

Application of the proposed "algorithmic staging" may provide a rational opportunity to tackle the complexity of mediastinal staging of lung cancer. The proposed protocol, on the basis of data provided by literature and of plain statistical concepts, may lead to a better use of available resources (in terms of costs/benefits). Although it may be generally accepted that if irrational use of limited resources is unbearable, we may as well consider that application of multiple (unnecessary) examinations may lead to ambiguity.

Nevertheless, the suggested protocol represents a simulation based on statistical projections and yet requires a validating study before implementation in clinical practice. 
Table 2. - Simplified Algorithm of mediastinal study. Red: percentage of positive or negative results depending on the pre-test probability considered. Blue: post-test probability of mediastinal involvement by the examinations performed

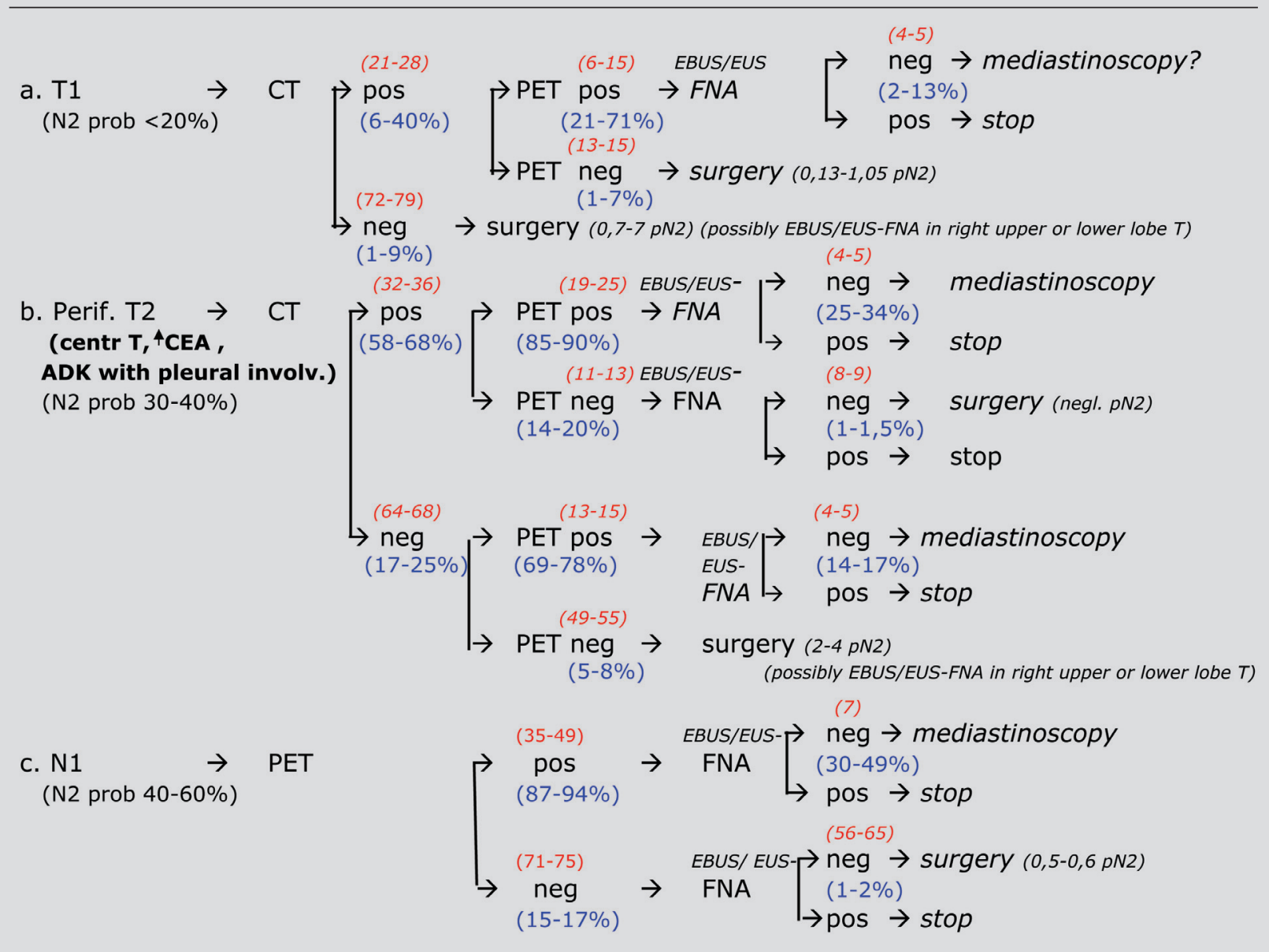

a. "Pre-test" probability of mediastinal metastasis $<20 \%$. CT negative mediastinum indicates probability of metastasis $<10 \%$; in this case, immediate surgery may be considered reasonable. Positive CT indicates probability of metastasis up to approx. 40\%; negative PET authorises surgery (1-7\% probability of mediastinal metastasis). Positive PET, followed by negative EBUS or EUS-FNA indicates reduction in N2 probability to $2-13 \%$; in this case, with reference to higher levels of "pretest" probability (T approx $3 \mathrm{~cm}$ ), confirmation by mediastinoscopy should be considered advisable.

b. "Pre-test" probability of mediastinal metastasis 30-40\%. Positive CT indicates probability of mediastinal metastasis about $58-68 \%$. Negative PET would reduce the probability of metastasis to no less than $14 \%$ (risk still too high to resort to surgery); therefore, Ultra Sound Guided Needle Aspiration is the best choice. Otherwise, negative CT followed by negative PET clears way to surgery. Negative CT, followed by positive PET, negative EBUS and EUS-FNA, requires confirmation by mediastinoscopy (probability reduced to $14-17 \%$ ).

c. "Pre-test" probability of mediastinal metastasis 40-60\%. Negative PET does not exclude (with sufficient safety margin) the presence of mediastinal metastasis (probability 12-17\%). Cytologic study of mediastinum must therefore be performed regardless of PET result (which may anyway follow for evaluation of distant metastasis).

\section{References}

1. Arita T, Matsumoto T, Kuramitsu T, et al. Is it possible to differentiate malignant mediastinal nodes from benign nodes by size? Reevaluation by CT, transesophageal echocardiography, and nodal specimen. Chest 1996; 110; 1004-1008.

2. Kerr KM, Lamb D, Wathen CG, Walker WS, Douglas NJ. Pathological assessment of mediastinal lymph nodes in lung cancer: implications for non-invasive mediastinal staging. Thorax 1992; 47: 337-341.

3. Detterbeck FC, Jantz MA, Wallace M, Vansteenkiste J Silvestri GA. Invasive Mediastinal Staging of Lung Cancer. ACCP Evidence-Based Clinical Practice Guidelines (2nd Edition). Chest 2007; 132: 202S-220.
4. Vansteenkiste JF, Stroobants SG, De Leyn PR, et al. Mediastinal lymph node staging with FDG-PET scan in patients with potentially operable non-small cell lung cancer: a prospective analysis of 50 cases. Chest 1997; 112: $1480-1486$.

5. Poncelet AJ, Lonneux M, Coche E, Weynaud B, Nairhomme P. PET-FDG scan enhances but does not replace preoperative surgical staging in non-small cell lung carcinoma. Eur J Cardiothorac Surg 2001; 20: 468-474.

6. Farrel MA, McAdams HP, Herndon JE, Patz EF Jr. Non-small cell lung cancer: FDG-PET for nodal staging in patients with stage I disease. Radiology 2000; 215: 886-890.

7. Gould MK, Kuschner WG, Rydzak CE, et al. Test Performance of Positron Emission Tomography and Com- 
puted Tomography for Mediastinal Staging in Patients with Non-Small-Cell Lung Cancer. A Meta-Analysis. Ann Intern Med 2003; 139: 879-892.

8. Miller DL, Rowland CM, Deschamps C, Allen MS, Trastek VF, Pairolero PC. Surgical treatment of nonsmall cell lung cancer $1 \mathrm{~cm}$ or less in diameter. Ann Thorac Surg 2002; 73: 1545-1551.

9. Watanabe S, Asamura H, Suzuki K, Tsuchiya R. Problems in diagnosis and surgical management of clinical N1 non-small cell lung cancer. Ann Thorac Surg 2005; 79: 1682-1685.

10. Ceron L, Mazzini R, Michieletto L, Pagan V, Serena P, Zamperlin A. Proposta di utilizzo razionale della tomografia a emissione di positroni (PET) nella diagnosi e stadiazione del tumore polmonare non a piccole cellule. Rassegna di Patologia dell'Apparato Respiratorio 2007; 22: 43-53.

11. Leyn PD, Lardinois D, Van Schil PE, et al. ESTS guidelines for preoperative lymph node staging for nonsmall cell lung cancer. Eur J Cardiothorac Surg 2007; 32: 1-8.

12. Behzadi A, Ung Y, Lowe V, Deschamps C. The role of positron emission tomography in the management of non-small cell lung cancer. Can J Surg 2009; 52: 235242.

13. Harrow EM, Abi-Saleh W, Blum J, et al. The Utility of Transbronchial Needle Aspiration in the Staging of Bronchogenic Carcinoma. Am J Respir Crit Care Med 2000; 161: 601-607.

14. Toloza EM, Harpole L, Detterbeck F, McCrory DC. Invasive Staging of Non-small Cell Lung Cancer. Chest 2003; 123: 157S-166S

15. Herth F, Becker HD, Ernst A. Ultrasound-Guided Transbronchial Needle Aspiration. An Experience in 242 Patients. Chest 2003; 123: 604-607.

16. Silvestri GA, Hoffman BJ, Bhutani MS, et al. Endoscopic ultrasound with fine-needle aspiration in the diagnosis and staging of lung cancer. Ann Thorac Surg 1996; 61: 1441-1445.

17. Herth F, Becker HD, Ernst A. Conventional vs Endobronchial Ultrasound-Guided Transbronchial Needle Aspiration. Chest 2004; 125: 322-325.

18. Herth FJ, Eberhardt R, Vilmann P, Krasnik M, Ernst A. Real-time endobronchial ultrasound guided transbronchial needle aspiration for sampling mediastinal lymph nodes. Thorax 2006; 61: 795-798.

19. Kim E, Telford JJ. Endoscopic ultrasound advances, part 1: Diagnosis. Can J Gastroenterol 2009; 23: 594601.
20. Trisolini R, Lazzari Agli L, Patelli M. Conventional vs Endobronchial Ultrasound-Guided Transbronchial Needle Aspiration of the Mediastinum. Chest 2004; 126; 1005-1006.

21. Shannon JJ, Bude RO, Orens JB, et al. Endobronchial ultrasound-guided needle aspiration of mediastinal adenopathy. Am J Respir Crit Care Med 1996; 153 (4 Pt 1): 1424-1430.

22. Fritscher-Ravens A. Endoscopic ultrasound evaluation in the diagnosis and staging of lung cancer. Lung Cancer 2003; 41: 259-267.

23. Micames CG, McCrory DC, Pavey DA, Jowell PS, Gress FG. Endoscopic Ultrasound-Guided Fine-Needle Aspiration for Non-small Cell Lung Cancer Staging. Chest 2007; 131; 539-548.

24. Gomez M, Silvestri GA. Endobronchial Ultrasound for the Diagnosis and Staging of Lung Cancer. Proc Am Thorac Soc 2009; 6: 180-186.

25. Vilmann P, Puri R. The complete "medical" mediastinoscopy (EUS-FNA + EBUS-TBNA). Minerva Med 2007; 98: 331-338.

26. Hishida T, Yoshida J, Nishimura M, Nishiwaki Y, Nagai $\mathrm{K}$. Problems in the current predictive standards of clinical N1 non-small cell lung cancer. Thorax 2008; 63: 526-531.

27. Cerfolio RJ, Bryant AS, Eloubeidi MA. Routine Mediastinoscopy and Esophageal Ultrasound Fine-Needle Aspiration in Patients With Non-small Cell Lung Cancer Who Are Clinically N2 Negative. Chest 2006; 130: 1791-1795.

28. Meyers BF, Haddad F, Siegel BA, et al. Cost-effectiveness of routine mediastinoscopy in computed tomography- and positron emission tomography-screened patients with stage I lung cancer. $J$ Thorac Cardiovasc Surg 2006; 131: 822-829.

29. Takamochi K, Nagai K, Suzuki K, Yoshida J, Ohde Y, Nishiwaki Y. Clinical predictors of N2 disease in nonsmall cell lung cancer. Chest 2000; 117: 1577-1582.

30. Takizawa T, Terashima M, Koike T, et al. Lymph node metastasis in small peripheral adenocarcinoma of the lung. J Thorac Cardiovasc Surg 998; 116: 276-280.

31. Buy JN, Ghossain MA, Poirson F, et al. Computed tomography of mediastinal lymph nodes in nonsmall cell lung cancer. A new approach based on the lymphatic pathway of tumor spread. J Comput Assist Tomogr 1988; 12: 545-552.

32. Cerfolio RJ, Bryant AS. Distribution and likelihood of lymph node metastasiss based on the lobar location of nonsmall-cell lung cancer. Ann Thorac Surg 2006; 81: 1969-1973.

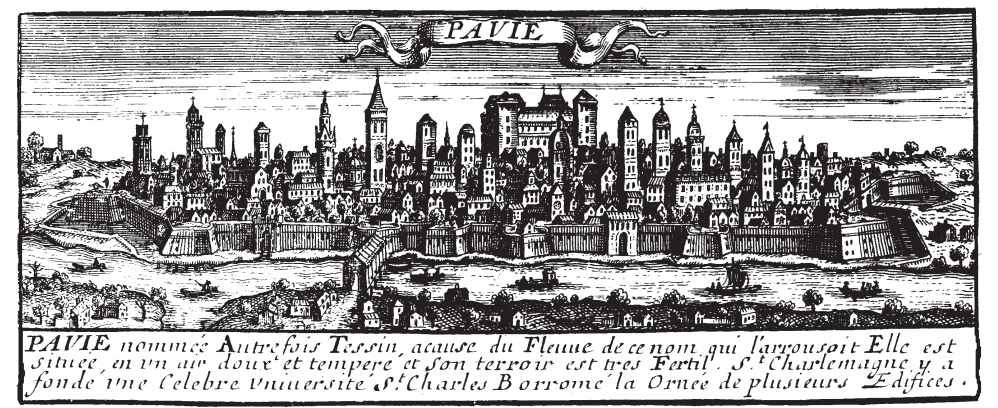

